\title{
Caracterização de pinos da blenda poli(L-Co-D,L ácido láctico)/poli(caprolactona triol) (PLDLA/PCL-T) e análise das propriedade mecânicas dos pinos durante degradação in vitro
}

\section{Characterization of pin blends Poly (L-Co-D,L lactic acid) /Poly(caprolactone diol) (PLDLA/PCL-T) and analysis of mechanical property of pins during in vitro degradation}

\author{
Marcia Adriana Tomaz Duarte ${ }^{1,2}$, Adriana Cristina Motta ${ }^{1}$ e Eliana Aparecida de Rezende Duek ${ }^{1,3}$ \\ 'Departamento de Ciências Fisiológicas, Centro de Ciências Médicas e Biológicas, \\ Pontifícia Universidade Católica de São Paulo - PUC-SP, Sorocaba, SP, Brasil \\ ${ }^{2}$ Mestrado Profissional em Engenharia Mecânica, Centro Universitário SOCIESC, \\ Santa Catarina, SC, Brasil \\ ${ }^{3}$ Departamento de Engenharia de Materiais, Faculdade de Engenharia Mecânica, \\ Universidade Estadual de Campinas - UNICAMP, Campinas, SP, Brasil
}

*eliduek@fem.unicamp.br

\begin{abstract}
Resumo
Os dispositivos de fixação óssea, metálicos convencionais, usados em cirurgia crâniomaxilofacial têm apresentado alguns problemas, tais como, corrosão, inflamação e infecção, além de neoformação de estrutura óssea mecanicamente inferior devido à atrofia gerada pela diferença de módulo elástico entre metal e osso, razões que têm levado ao aumento do interesse por dispositivos poliméricos bioarreabsorvíveis. Os polímeros biorreabsorvíveis mais utilizados nesta aplicação pertencem à família dos poli ( $\alpha$-hidroxi ácidos), que têm como característica degradarem por hidrólise de suas ligações ésteres, tal como copolímero poli (L-ácido láctico-co-D, L ácido láctico), PLDLA. Neste trabalho foram investigados alguns efeitos da adição de poli (caprolactona triol), PCL-T sobre PLDLA. Foram preparados pinos por fusão de blendas nas seguintes composições 100/0, 90/10, 70/30 and 50/50 (m/m), PLDLA/PCL-T. Os pinos foram caracterizados por diferentes técnicas (DSC, MEV e ensaio mecânico). A degradação in vitro dos pinos foi investigada, sendo observado que a adição de PCL-T no PLDLA modificou suas propriedades mecânicas e morfológicas. Tais mudanças podem apresentar potencial para outras aplicações do material, onde a questão da flexibilidade se faça necessária.
\end{abstract}

Palavras-chaves: $P L D L A / P C L-T$, caracterização, propriedades mecânicas, in vitro.

\begin{abstract}
The bone fixation devices, conventional metallic cranium used in surgery have presented some problems, such as corrosion, inflammation and infection, and a lower mechanically newly formed bone structure due to the atrophy caused by the difference in stiffness between metal and bone. These reasons have led to increased interest in bioreabsorbable polymeric devices. The most bioresorbable polymers used in this application belong to the family of poly ( $\alpha$-hydroxy acids), which are characterized degrade by hydrolysis of its ester linkages, such as copolymer poly (L-lactic acid-co-D, L lactic acid) (PLDLA). In this work was investigated some effects of the addition poly (caprolactone triol), PCL-T in the PLDLA. Pins were prepared by melting in the 100/0, 90/10, 70/30 and 50/50 (w/w) compositions, PLDLA/PCL-T. The pins were characterized by different methods (DSC, SEM and mechanical test). The in vitro pins degradation was investigated. It was observed that the addition of PCL-T of PLDLA modifies the mechanical properties, morphological, we conclude that PLDLA/PCL-T can be a potential material for various applications, where the question of flexibility to make necessary.
\end{abstract}

Keywords: PLDLA/PCL-T, characterization, mechanical properties, in vitro. 


\section{Introdução}

Os defeitos ósseos podem ser resultado de anormalidades congênitas, traumas ou doenças. As aplicações de dispositivos de fixação óssea interna a partir de biomateriais, utilizados nestas situações, revolucionaram o campo de consolidação de fraturas ósseas. Isto se deve às novas tecnologias disponíveis, resultantes do desenvolvimento de materiais que geram produtos que atendem às solicitações para uso biomédico.

A seleção do material é importante e deve garantir biocompatibilidade e resistência à corrosão durante o tempo necessário para recuperação do osso ${ }^{[1]}$ e embora os materiais metálicos permitam bons resultados clínicos através de uma fixação estável do osso, seu emprego esta associado a algumas limitações e riscos, como a possibilidade de re-fratura ao longo do osso no local onde estava presente o dispositivo ${ }^{[2,3]}$ em função da grande diferença de rigidez entre o osso $(E=1-30 \mathrm{GPa})$ e os metais $(E=100-200 \mathrm{GPa})$, ou ainda a possibilidade de reações inflamatórias por parte do tecido circunjacente ao implante metálico ${ }^{[4]}$, causadas pela presença do metal por um longo período no corpo.

Dessa forma, os dispositivos metálicos, em inúmeras situações, devem ser removidos, por meio de uma $2^{\circ}$ intervenção cirúrgica corroborando com a ideia de que não representam portanto os biomateriais ideais para aplicações ortopédicas, em função de reações alérgicas e liberação de íons metálicos ao redor do tecido implantado ${ }^{[5]}$.

Neste contexto os poliméricos biorreabsorvíveis tem sido uma classe de materiais atrativa nas últimas décadas, sendo utilizados e experimentados em vários aspectos das cirurgias ortopédicas, incluindo fixações de fraturas, reposição óssea, reparo da cartilagem, reparo do menisco e fixação de ligamentos ${ }^{[6,7]}$. Esses materiais biorreabsorvíveis têm sido utilizados na forma de parafusos, pinos e placas nas aplicações ortopédicas e cirurgias orais em humanos e animais ${ }^{[8]}$.

O implante de materiais poliméricos biorreabsorvíveis suporta uma elevada fração de carga transmitida ao osso durante os primeiros estágios do processo de recuperação. A redução gradual da resistência do implante, devido à sua reabsorção, transfere uma porcentagem crescente da carga ao osso em recuperação. Como resultado, o local da fratura recuperado desenvolve uma resistência comparável àquela original do osso ${ }^{[9]}$.

Os poli ( $\alpha$-hidróxi ácidos) são considerados uma das famílias de polímeros mais promissoras na área dos bioreabsorvíveis. A grande vantagem desses polímeros está na sua forma de degradação que ocorre por hidrólise de suas ligações ésteres, sendo os produtos gerados completamente absorvidos pelo organismo. Dentre esses polímeros destaca-se o poli (L-ácido láctico-co-D, Lácido láctico) (PLDLA), em função do adequado tempo de degradação que apresenta, compatível ao requerido na fixação de fraturas ósseas.

O estudo tem caracterizado a retenção de resistência durante a degradação do copolímero PLDLA (70/30), que mantem $90 \%$ da resistência após 6 meses, $70 \%$ da resistência após 9 meses, 50\% da resistência após 12 meses e nenhuma resistência após 18 meses $^{[10]}$. A tecnica de auto-reforço inventada e patenteada por Törmälä e colaboradores ${ }^{[11]}$, onde tanto a matriz quanto o elemento reforçante são do mesmo polímero permite a obtenção de dispositivos poliméricos maleáveis e resistentes, sendo diversos os estudos de auto- reforço ao PLDLA.

Uma grande vantagem dessa técnica é a possibilidade da manufatura de dispositivos com tamanhos menores que podem ser flexionadas a frio. Comercialmente os copolímeros auto-reforçados PLDLA (70/30) (placas e parafusos) são bastante empregados (Biosorb FX, Bionximplants LTD, Tampere, Finlândia). Entretanto a técnica exige que sejam realizadas diversas etapas de processamento, o que representa uma dificuldade na obtenção do produto final.

Blendas de PLDLA com PCL-triol têm sido alvo de estudo na busca por aliar as características mecânicas do PLDLA com a flexibilidade do PCL triol. O PCL-triol age como um plastificante, enfraquecendo as forças intermoleculares entre as cadeias poliméricas ${ }^{[12]}$, com objetivo de melhorar a processabilidade e aumentar a flexibilidade. Essa característica que o PCL-T atribui ao PLDLA em relação à flexibilidade pode ser interessante em diversas aplicações, como por exemplo, na engenharia tecidual. Espósito ${ }^{[13]}$ realizou um estudo no qual a blenda PLDLA/PCL-T servia como scaffolds para crescimento celular visando aplicação em regiões como menisco.

Com isto, o objetivo deste estudo foi obter materiais que atendam a solicitação mecânica para fixação óssea sem a necessidade de auto-reforço, preparando blendas de PLDLA com PCL-triol em diversas proporções, sendo avaliadas as características da blenda. Foi acompanhado, o perfil da degradação in vitro.

\section{Parte Experimental}

\subsection{Materiais}

O poli (L-co-DL, ácido lático) (70:30) PLDLA, foi sintetizado nos laboratório da PUC/ Sorocaba, com massa molar média de 265000g.mol ${ }^{-1}$ de acordo com Motta ${ }^{[14]}$. A poli (caprolactona triol), (PCL-T), cujo nome químico é 2-oxepanona, foi fornecida pela Solvay (CAPA 3091), com massa molar média de $900 \mathrm{~g} \cdot \mathrm{mol}^{-1}$.

\subsection{Preparação dos pinos por fusão}

Foram preparadas blendas de PLDLA/PCL-T por fusão, obtendo-se pinos com $2 \mathrm{~mm}$ de diâmetro, nas proporções 100/0,90/10,70/30,50/50\% (m/m), utilizando uma mini-injetora Mini Max Molder modelo LMM - 2017. (Figura 1)

As amostras foram colocadas na mini injetora, e aquecidas a $200^{\circ} \mathrm{C}$ por 1 minuto sem cisalhamento, seguido de $1,5 \mathrm{~min}$ com cisalhamento (velocidade de cisalhamento constante, de $4 \mathrm{rpm}$ ). O molde foi envolto por uma camisa para mantê-lo aquecido a $110^{\circ} \mathrm{C}$. Após esse procedimento a mistura foi injetada no molde, deixado esfriar a temperatura ambiente. Depois de retirado do molde o pino foi armazenado no dessecador.

\subsection{Caracterização do PLDLA/PCL-T obtido por fusão}

Calorimetria exploratória diferencial (DSC): As análises de DSC foram realizadas em um equipamento modelo 2920 da TA Instruments. Amostras pesando aproximadamente $7-10 \mathrm{mg}$ foram aquecidas de $25{ }^{\circ} \mathrm{C}$ a $200{ }^{\circ} \mathrm{C}$ a uma taxa 
de aquecimento de $10^{\circ} \mathrm{C} \cdot \mathrm{min}^{-1}$ (primeiro aquecimento), e mantidas a esta temperatura por $5 \mathrm{~min}$. Subsequentemente foram resfriadas a $-100{ }^{\circ} \mathrm{C}$ a uma taxa de $10{ }^{\circ} \mathrm{C} \cdot \mathrm{min}^{-1}$ e mantidas nessa temperatura por $5 \mathrm{~min}$. Após, foram aquecidas novamente ate $200^{\circ} \mathrm{C}$ a uma taxa de $10{ }^{\circ} \mathrm{C} / \mathrm{min}$ sob atmosfera de nitrogênio.

Microscopia eletrônica de varredura (MEV): As amostras das superfícies superiores e das fraturas (obtidas em nitrogênio líquido) dos pinos foram metalizadas com ouro (Sputter Coater BAL-TEC SCD 050) e analisadas em um microscópio eletrônico de varredura (JEOL JXA 860) operado a $10 \mathrm{kV}$.

Ensaio mecânico de Flexão: Os pinos de PLDLA/PCL-T nas composições $(100 / 0),(90 / 10),(70 / 30),(50 / 50) \%(\mathrm{~m} / \mathrm{m})$ com cerca de $1,94( \pm 0,45) \mathrm{mm}$ de diâmetro foram submetidos a ensaios de flexão em equipamento servo-hidráulico da Testar II (modelo 810 MTS), célula de carga com capacidade de $1 \mathrm{kN}$ e veloc.50 mm. min ${ }^{-1}$ com vão de $30 \mathrm{~mm}$. Foram ensaiados 5 corpos de prova de cada amostra nas mesmas condições de umidade $(50 \%)$ e temperatura $\left(25^{\circ} \mathrm{C} \pm 2\right)$.

\subsection{Degradação in vitro - análise macroscópica e comportamento mecânico dos pinos PLDLA/PCL-T}

Os pinos de PLDLA/PCL-T nas composições 100/0, 90/10, 70/30 e 50/50 foram colocados em tubos de ensaios com tampa rosqueada, previamente esterilizados contendo solução tampão fosfato (PBS) $\mathrm{pH} 7,4$ a $37 \pm 1{ }^{\circ} \mathrm{C}$, sendo retirados após 4, 8, 12 e 24 semanas, lavados com água destilada e secos sob vácuo, durante 48 horas. Depois de secas as amostras foram caracterizadas pelas técnicas descritas a seguir:

Ensaio mecânico de Flexão: Os pinos de PLDLA/PCL-T nas composições $(100 / 0),(90 / 10),(70 / 30),(50 / 50) \%(\mathrm{~m} / \mathrm{m})$ com cerca de $1,94( \pm 0,45) \mathrm{mm}$ de diâmetro foram submetidas a ensaios de flexão em um equipamento servohidráulico da Testar II, modelo 810, do fabricante MTS, célula de carga com capacidade de $1 \mathrm{kN}$ e velocidade de $50 \mathrm{~mm} . \mathrm{min}^{-1}$ com vão de $30 \mathrm{~mm}$. As amostras foram submetidas a 5 ensaios nas mesmas condições de umidade $(50 \%)$ e temperatura $\left(25^{\circ} \mathrm{C} \pm 2\right)$.

Microscopia eletrônica de varredura (MEV): Foi acompanhado por meio da analises de MEV o processo de degradação dos pinos.

Avaliação Macroscópica: Foi realizada a avaliação macroscópica das amostras em função do tempo de imersão na solução tampão fosfato para as diferentes composições de PLDLA/PCL-T.

\section{Resultados}

\subsection{Caracterização das composições PLDLA-PCL-triol}

Calorimetria exploratória diferencial (DSC): Os resultados referentes ao DSC do sistema PLDLA/PCL-T nas composições $100 / 0,90 / 10,70 / 30$ e 50/50 estão sumarizados na Tabela 1 .

As análises de DSC conforme Tabela 1 mostram as temperatura de transição vítrea $\left(\mathrm{T}_{\mathrm{g}}\right)$,para os polímeros porém, apenas o PCL-T apresenta temperatura de fusão $\left(\mathrm{T}_{\mathrm{m}}\right)$,
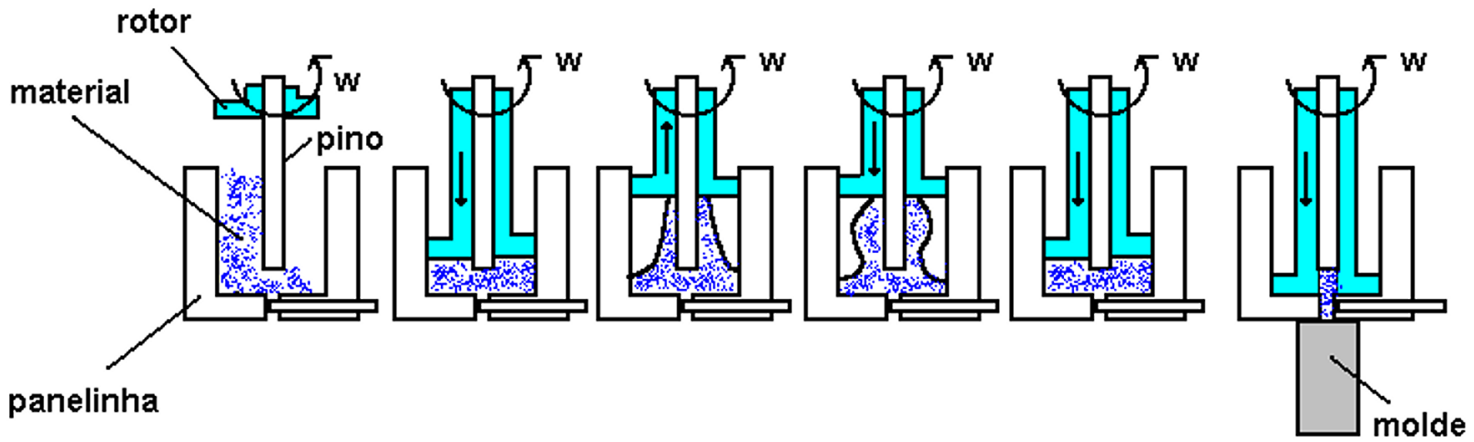

Figura 1. Esquema de funcionamento da mini injetora Mini Max Moulder ${ }^{[15]}$.

Tabela 1. Resultados da analise de DSC para as várias composições PLDLA-PCL-triol.

\begin{tabular}{|c|c|c|c|c|c|c|c|}
\hline $\begin{array}{c}\text { Composição } \\
\text { (PLDLA/PCL-T) }\end{array}$ & Aquecimento & $\begin{array}{c}\mathrm{T}_{\mathrm{g}} \\
\text { PCL-T } \\
\left({ }^{\circ} \mathrm{C}\right)\end{array}$ & $\begin{array}{c}\mathbf{T}_{\mathbf{c}} \\
\text { PCL-T } \\
\left({ }^{\circ} \mathrm{C}\right)\end{array}$ & $\begin{array}{c}\Delta \mathrm{H}_{\mathrm{c}} \\
\text { PCL-T } \\
(\mathrm{J} / \mathrm{g})\end{array}$ & $\begin{array}{c}\mathbf{T}_{\mathrm{m}} \\
\text { PCL-T } \\
\left({ }^{\circ} \mathrm{C}\right)\end{array}$ & $\begin{array}{c}\Delta H_{\mathrm{m}} \\
\text { PCL-T } \\
(\mathrm{J} / \mathrm{g})\end{array}$ & $\begin{array}{c}\text { Tg } \\
\text { PLDLA } \\
\left({ }^{\circ} \mathrm{C}\right)\end{array}$ \\
\hline $100 / 0$ & $1^{\circ}$ & - & - & - & - & - & 57 \\
\hline $100 / 0$ & $2^{\circ}$ & - & - & - & - & - & 57 \\
\hline $90 / 10$ & $1^{\circ}$ & - & - & - & - & - & 42 \\
\hline $90 / 10$ & $2^{\circ}$ & -67 & - & - & - & - & 48 \\
\hline $70 / 30$ & $1^{\circ}$ & - & - & - & - & - & 40 \\
\hline $70 / 30$ & $2^{\circ}$ & -73 & -32 & 2 & 1 & 1 & 48 \\
\hline $50 / 50$ & $1^{\circ}$ & - & - & - & - & - & 41 \\
\hline $50 / 50$ & $2^{\circ}$ & -67 & -32 & 2 & 3,4 & 0,5 & 46 \\
\hline
\end{tabular}


caracterizando o PLDLA como polímero amorfo. O pino de PLDLA puro mostrou uma $\mathrm{Tg}$ de $57^{\circ} \mathrm{C}$ no primeiro e segundo aquecimento conforme Figura 2a, $\mathrm{b}$.

A adição de 10\% de PCL-T diminuiu a Tg do PLDLA, de $57^{\circ} \mathrm{C}$ para $48^{\circ} \mathrm{C}$, sendo que as demais composições de PCL-T mantiveram esse mesmo patamar de diminuição, independente da quantidade de PCL-T ser maior.

Concentração do PCL-T maiores que $10 \%$, não modificaram significativamente o comportamento térmico do PLDLA, o que pode caracterizar como sendo esse um valor limite de concentração do PCL-T, acima do qual não influencia nas propriedades térmicas desse material conforme observado na Tabela 1.

\subsection{MEV - Microscopia eletrônica de varredura (Pinos)}

A Figura 3 mostra as micrografias obtidas por MEV das topografias das superfícies e das superfícies de fratura do PLDLA/PCL-T em várias composições obtido por fusão. O copolímero PLDLA em forma de pino apresenta uma topografia de superfície densa, lisa apresentando algumas incrustações (Figura 3a) provenientes do processo de fusão do material. Na análise microscópica da superfície de fratura é possível observar um material compacto, denso com ausência de poros (Figura 3b).

A composição 90/10 apresentou uma morfologia de superfície similar ao copolímero puro, conforme demonstrado na Figura 3c. A observação da fratura nesta composição

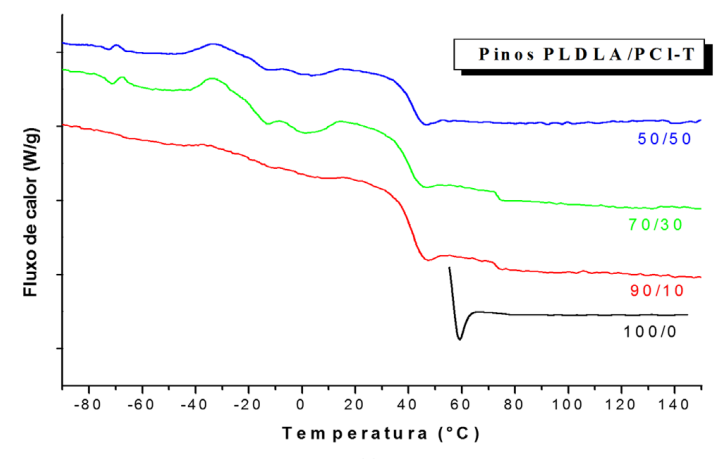

(a)

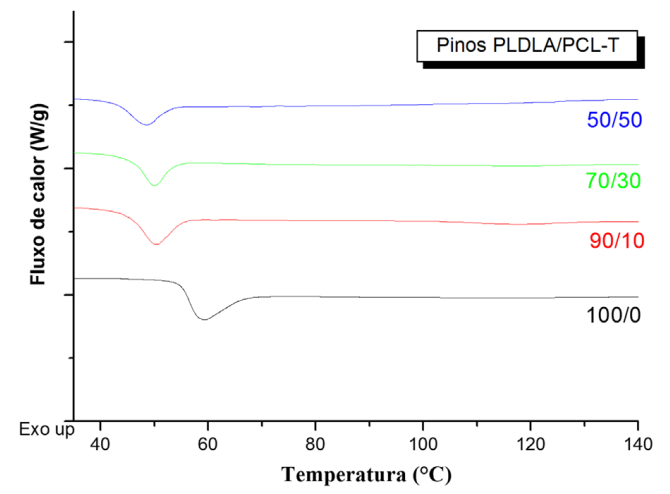

(b)

Figura 2. Termogramas de DSC para o sistema PLDLA/PCL-T obtidas por fusão. (a) primeiro aquecimento; (b) segundo aquecimento. evidenciou a presença de poros, Figura 3d. Os poros estavam distribuídos homogeneamente e apresentavam diâmetros de 0,75-1 $\mu \mathrm{m}$, em formato esférico.

Os pinos com composição 70/30 (Figura 3e, f) e 50/50 (Figura $3 \mathrm{~g}, \mathrm{~h}$ ) tem topografia de superfície e superfície de fratura similares, o que pode estar associado ao valor limite de solubilidade. Em relação à composição $90 / 10$ os pinos $70 / 30$ e 50/50 apresentaram maior quantidade de poros em toda a amostra com uma morfologia esférica. Esse fato também foi observado por DSC onde não houve alteração significativa adicionando 30 e $50 \%$ do PCL-T.

A presença dos poros foi atribuída ao efeito plastificante do PCL-T, pois este foi o único diferencial na confecção dos pinos para origem dos poros.

Luciano $^{[16]} \mathrm{em}$ seu estudo com membranas obtidas por evaporação de solvente observou que a adição do plastificante tri-etil-citrato na membrana densa tornava-a porosa. Segundo o autor o plastificante facilita a mobilidade das cadeias que se organizam ao redor de núcleos de cristalização já presente no polímero (semicristalino) e ocorre o surgimento de glóbulos. O aglomerado desses glóbulos gera uma estrutura porosa. Duarte ${ }^{[17]}$ ao estudar de PLDLA/PCL-T na forma de membranas já havia verificado que a presença do PCL-T modificava a membrana de PLDLA tornando-a porosa.

Outra possível explicação para formação desses poros, baseado nos resultados, é que em função da presença de PCL-T provocar uma maior mobilidade nas cadeias, e estas por sua vez, na tentativa de se organizarem na nova condição que se encontram permitem a geração de pequenos espaços na estrutura, já que a distribuição aleatória das unidades levógiro e dextrógero, dificulta o processo de cristalização.

\subsection{Ensaio de flexão a 3 pontos}

O ensaio de flexão a 3 pontos mostrou que os pinos PLDLA/PCL-T obtidos por fusão têm características de um material dúctil.

$\mathrm{Na}$ Figura 4, pode se observar que a presença do PCL-T modificou o módulo de elasticidade do material. Essa mudança do módulo de elasticidade pode representar um ganho em possibilidade de aplicações da blenda.

A Tabela 2 mostra os dados obtidos do gráfico.

\subsection{Análise in vitro dos pinos de PLDLA/PCL-T}

$\mathrm{O}$ ensaio de flexão a 3 pontos mostrou como os pinos PLDLA/PCL-T se comportam num ambiente que simula as condições corpóreas, e a Tabela 3, apresenta esses dados.

Analisando a Tabela 3 , pode se verificar que diferentemente do PLDLA puro, que durante o processo de degradação apresenta um aumento no modulo de elasticidade, para as demais situações em que temos as blendas, ocorre uma queda desse modulo de elasticidade no transcorrer dos dias, ainda que seja verificado em algumas composições, um aumento inicial deste parâmetro. Essa situação, de aumento do módulo de elasticidade no inicio do processo de degradação, é observada em inúmeros trabalhos ${ }^{[18-20]}$, o que pode estar associado, por exemplo, a mudanças na massa molar do material. 


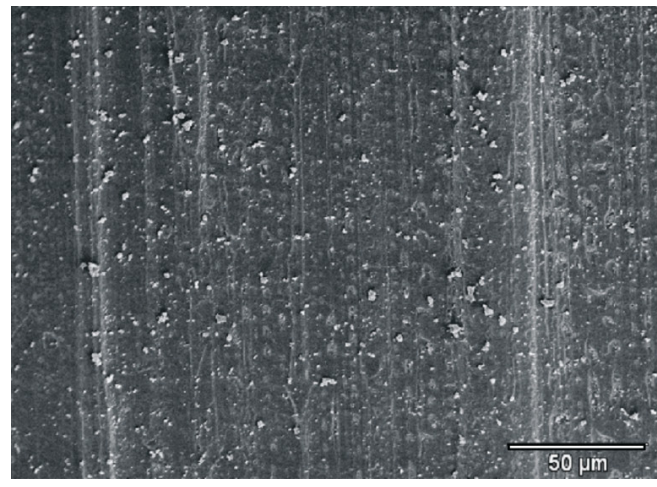

(a)

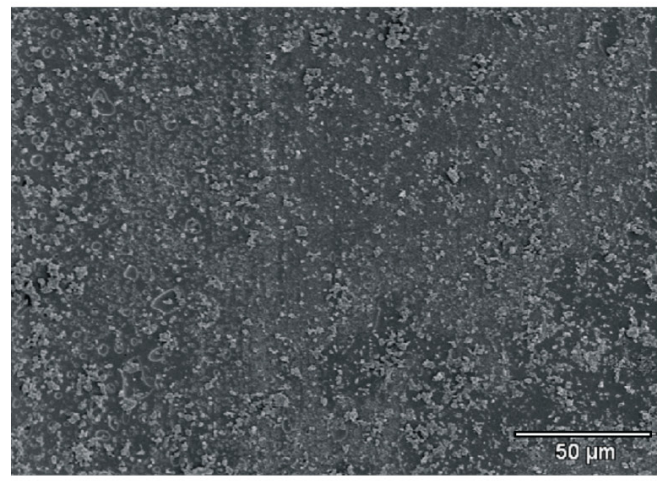

(c)

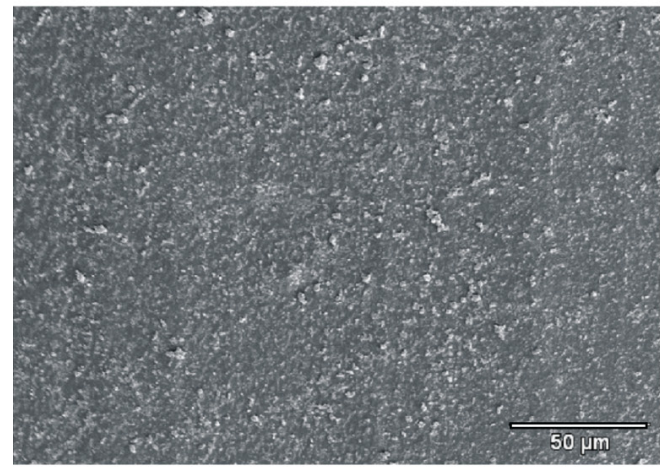

(e)

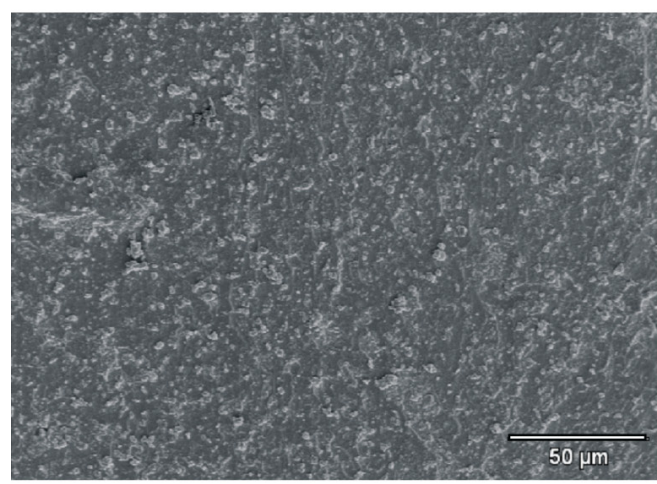

(g)

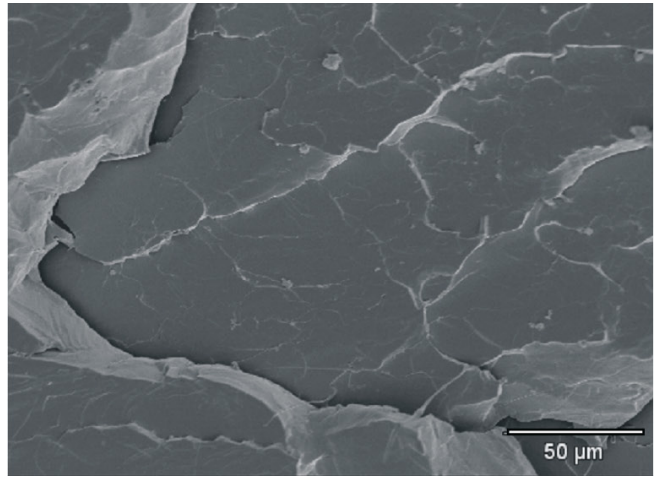

(b)

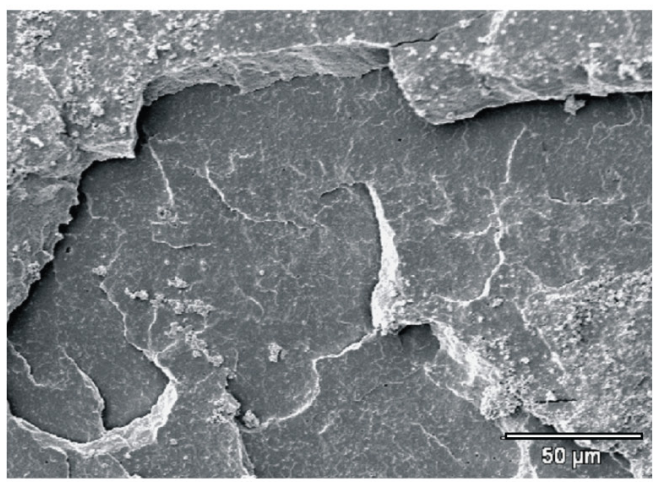

(d)

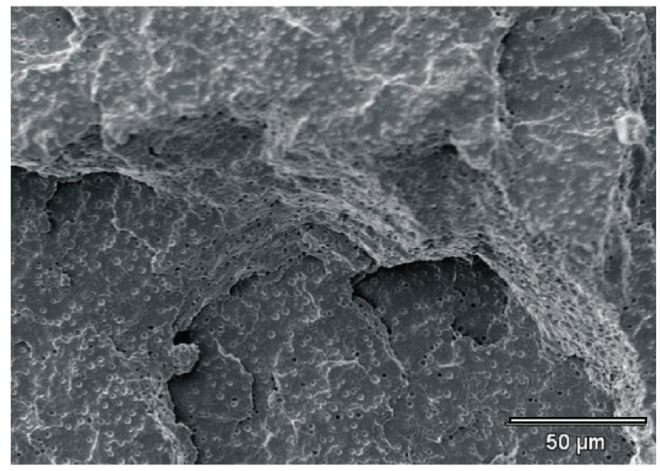

(f)

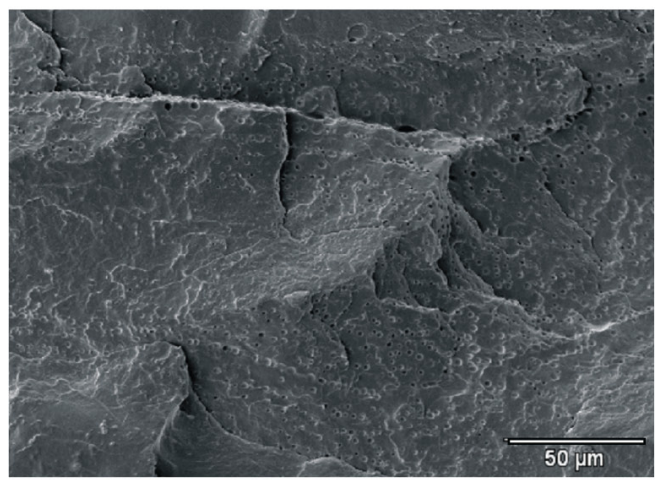

(h)

Figura 3. Micrografias de MEV da superfície (a) e de fratura (b) dos pinos PLDLA/PCL-T (100/0); da superficie (c) e de (d) fratura PLDLA/PCL-triol (90/10); da superfície (e) e de fratura (f) PLDLA/PCL-triol (70/30); da superfície (g) e de fratura (h) PLDLA/PCL-triol (50/50). 
Foi verificado que quanto maior a quantidade de PCL-T na blenda, menor era o módulo elástico. Para o tempo de 24 semanas, as blendas, nas diferentes composições, apresentavam- se incapazes de serem submetidas ao ensaio mecânico, evidenciando o processo de degradação que se encontravam. O perfil de degradação que cada composição da blenda apresenta, acompanhado pelos dados. É importante salientar que a escolha de uma blenda, para uma determinada aplicação dependerá de sua capacidade em manter suas propriedades por um tempo determinado.

Tabela 2. Valores de força máxima, módulo de elasticidade, tensão máxima e alongamento obtidos a partir do ensaio de flexão para os pinos PLDLA/PCL-T.

\begin{tabular}{|c|c|c|c|c|}
\hline $\begin{array}{c}\text { Composição: } \\
\text { PLDLA/ } \\
\text { PCL-T }\end{array}$ & $\begin{array}{c}\text { Força } \\
\text { Máxima } \\
\text { (N) }\end{array}$ & $\begin{array}{c}\text { Módulo de } \\
\text { Elasticidade } \\
\text { (E) } \\
\text { (MPa) }\end{array}$ & $\begin{array}{l}\text { Tensão } \\
\text { (MPa) }\end{array}$ & Alongamento \\
\hline $100 / 0$ & $11,8( \pm 0,1)$ & $3133( \pm 200)$ & $49,9( \pm 2,6)$ & $0,012( \pm 0,003)$ \\
\hline $90 / 10$ & $8.9( \pm 0,2)$ & $3212( \pm 200)$ & $47,9( \pm 0,01)$ & $0,016( \pm 0,0007)$ \\
\hline $70 / 30$ & $7,0( \pm 0,5)$ & $2525( \pm 290)$ & $43,4( \pm 3,0)$ & $0,017( \pm 0,001)$ \\
\hline $50 / 50$ & $4,4( \pm 0,9)$ & $1738( \pm 132)$ & $26( \pm 4)$ & $0,0149( \pm 0,001)$ \\
\hline
\end{tabular}

Tabela 3. Dados obtidos do ensaio de flexão nas diversas composições do PLDLA/PCL-triol nos tempos de estudo.

\begin{tabular}{|c|c|c|c|c|}
\hline $\begin{array}{c}\text { PLDLA/ } \\
\text { PCL-T(100/0) }\end{array}$ & $\begin{array}{c}\text { Força } \\
\text { Máxima } \\
\text { (N) }\end{array}$ & $\begin{array}{c}\text { Módulo de } \\
\text { Elasticidade } \\
\text { (E) } \\
\text { (MPa) }\end{array}$ & $\begin{array}{c}\text { Tensão } \\
\text { máxima } \\
\text { (MPa) }\end{array}$ & $\begin{array}{c}\text { Alongamento } \\
\text { na ruptura } \\
(\%)\end{array}$ \\
\hline 0 semana & $11,8( \pm 0,1)$ & $3133( \pm 200)$ & $50( \pm 2)$ & $0,012( \pm 0,003)$ \\
\hline 4 semanas & $12,6( \pm 0,4)$ & $3462( \pm 843)$ & $67,8( \pm 1,8)$ & $0,02( \pm 0,004)$ \\
\hline 8 semanas & $11,5( \pm 0,6)$ & $3791( \pm 214)$ & $59,5( \pm 5,0)$ & $0,016( \pm 0,002)$ \\
\hline 12 semanas & $11,4( \pm 0,3)$ & $4024( \pm 428)$ & $72,3( \pm 5,7)$ & $0,018( \pm 0,002)$ \\
\hline 16 semanas & $9,9( \pm 0,9)$ & $3049( \pm 285)$ & $48,7( \pm 9,8)$ & $0,015( \pm 0,002)$ \\
\hline 24 semanas & $7,8( \pm 1,1)$ & $3582( \pm 367)$ & $38,6( \pm 4,9)$ & $0,010( \pm 0,001)$ \\
\hline \multicolumn{5}{|l|}{$\begin{array}{c}\text { PLDLA/ } \\
\text { PCL-T }(90 / 10)\end{array}$} \\
\hline 0 semana & $8.9( \pm 0.2)$ & $3212( \pm 200)$ & $47,9( \pm 0,01)$ & $0,016( \pm 0,0007)$ \\
\hline 4 semanas & $7,9( \pm 0,4)$ & $3310( \pm 238)$ & $49,3( \pm 5,0)$ & $0,06( \pm 0,079)$ \\
\hline 8 semanas & $6,9( \pm 0.1)$ & $3293( \pm 111)$ & $44( \pm 3)$ & $0,013( \pm 0,001)$ \\
\hline 12 semanas & $4,2( \pm 0.8)$ & $2046( \pm 552)$ & $26,59( \pm 6,47)$ & $0,013( \pm 0,003)$ \\
\hline 16 semanas & $3,7( \pm 0,2)$ & $2773( \pm 431)$ & $20,45( \pm 0,97)$ & $0,007( \pm 0,001)$ \\
\hline 24 semanas & $*$ & $*$ & $*$ & $*$ \\
\hline \multicolumn{5}{|l|}{$\begin{array}{c}\text { PLDLA/ } \\
\text { PCL-T (70/30) }\end{array}$} \\
\hline 0 semana & $7,0( \pm 0,5)$ & $2525( \pm 290)$ & $43,4( \pm 3,0)$ & $0,017( \pm 0,001)$ \\
\hline 4 semanas & $6,0( \pm 0,2)$ & $2239( \pm 204)$ & $38,3( \pm 1,5)$ & $0,017( \pm 0,0004)$ \\
\hline 8 semanas & $5,9( \pm 0,5)$ & $2894( \pm 398)$ & $37,6( \pm 5,3)$ & $0,013( \pm 0,00004)$ \\
\hline 12 semanas & $4,6( \pm 0,5)$ & $2452( \pm 911)$ & $20,9( \pm 5,0)$ & $0,0089( \pm 0,002)$ \\
\hline 16 semanas & $3,2( \pm 1,1)$ & $2149( \pm 586)$ & $14,4( \pm 4,7)$ & $0,006( \pm 0,0004)$ \\
\hline 24 semanas & $*$ & $*$ & $*$ & $*$ \\
\hline \multicolumn{5}{|l|}{$\begin{array}{c}\text { PLDLA/ } \\
\text { PCL-T(50/50) }\end{array}$} \\
\hline 0 semana & $4,4( \pm 0,9)$ & $1738( \pm 132)$ & $26( \pm 4)$ & $0,0149( \pm 0,001)$ \\
\hline 4 semanas & $5,5( \pm 0,3)$ & $2223( \pm 118)$ & $37( \pm 4)$ & $0,017( \pm 0,001)$ \\
\hline 8 semanas & $5,3( \pm 0,3)$ & $2246( \pm 247)$ & $31,1( \pm 2,8)$ & $0,014( \pm 0,002)$ \\
\hline 12 semanas & $4,3( \pm 0,7)$ & $2256( \pm 266)$ & $22( \pm 8)$ & $0,0092( \pm 0,002)$ \\
\hline 16 semanas & $3,2( \pm 0,4)$ & $1688( \pm 269)$ & $14,2( \pm 1,4)$ & $0,008( \pm 0,001)$ \\
\hline 24 semanas & $*$ & $*$ & $*$ & $*$ \\
\hline
\end{tabular}

*situação onde não foi possível fazer análise.

\subsection{Análise macroscópica dos pinos nos diferentes tempos}

A avaliação macroscópica da degradação dos pinos em função do tempo de imersão em tampão fosfato, para as diferentes composições de PLDLA/PCL-T, esta apresentada na Figura 5.

O PLDLA é um material amorfo e apresenta como característica ser transparente, conforme a composição 100/0 PLDLA, Figura 5a. Com a adição do PCL-T o material se torna levemente opaco, sendo tal opacidade bem visível na composição 50/50, Figura 5d. Após 24 semanas de degradação, verificou-se uma aparência completamente esbranquiçada

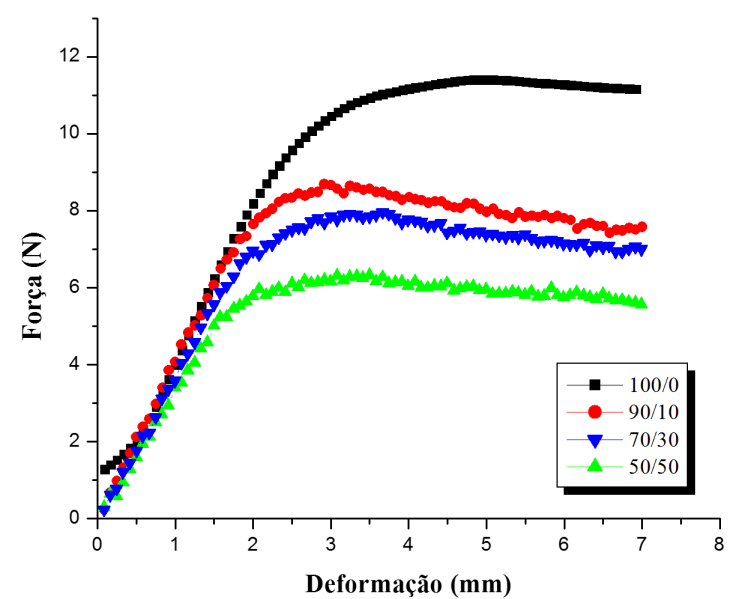

Figura 4. Curvas de força $x$ deformação das blendas PLDLA/PCL-T.
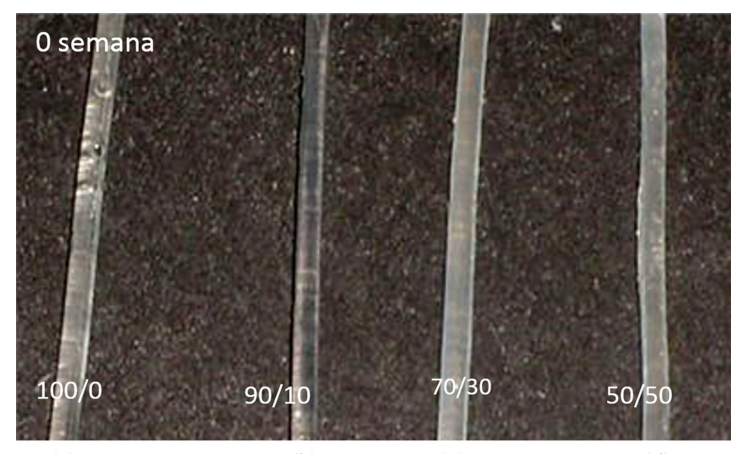

(a)

(b)

(c)

(d)

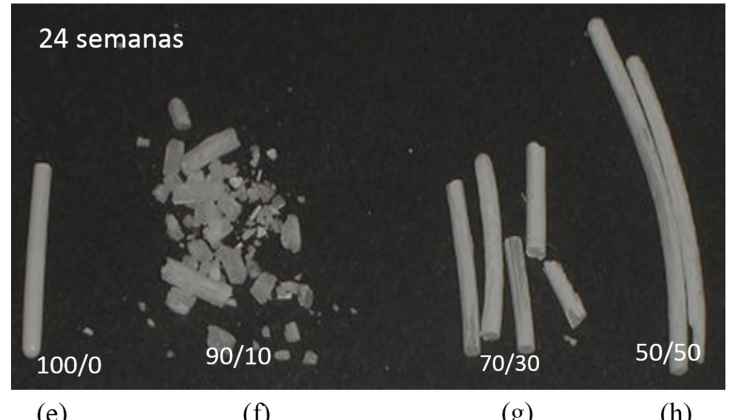

(e)

(f)

(g)

(h)

Figura 5. Avaliação macroscópica dos pinos antes e após degradação (0 semana e 24 semanas). 
para o PLDLA, Figura 5e, o que pode ser atribuído à recristalização do material. Em todas as composições era nítida a constatação do processo de degradação, transcorridos 24 semanas, no entanto, a composição que se apresentava com um processo de degradação mais intenso, apresentando-se até na forma de fragmentos do pino, foi a PLDLA/PCL-T 90/10, Figura 5f. justificando, portanto a impossibilidade notada de se fazer o ensaio mecânico, conforme Tabela 3.

\section{Conclusão}

Baseado nos resultados apresentados pôde-se verificar a influência da concentração do PCL-T nos pinos de PLDLA, o qual modificou suas propriedades mecânicas, térmicas, morfológicas. A análise de DSC mostrou que as Tg do PLDLA diminuem na presença do PCL-T blendas, e que essa diminuição se mostrou significativa com $10 \%$ de PCL-T, não sendo notada uma queda mais expressiva desse patamar para concentrações maiores de PCL-T na blenda. A análise de MEV mostrou o aparecimento de poros no PLDLA quando o PCL-T estava presente enquanto que o teste de flexão mostrou uma diminuição do módulo de elasticidade, quando a concentração de PCl-T aumentava na blenda Esse aumento na flexibilidade dos pinos PLDLA, causado pelo PCL-T pode representar um aumento do numero de aplicações do material, em especial na área de engenharia tecidual.

\section{Referências}

1. Cheung, H., Lau, K., Lu, T., \& Hui, D. (2007). A critical review on polymer-based bio-engineered materials for scaffold development. Composites: Part B, Engineering, 38(3), 291300. http://dx.doi.org/10.1016/j.compositesb.2006.06.014.

2. Feltel, R. M., Ahmed, I., Parsons, A. J., \& Rudd, C. D. (2013). Bioresorbable composite screws manufactured via forging process: pull-out, shear, flexural and degradation characteristics. Journal of the Mechanical Behavior of Biomedical Materials, 18, 108-122. http://dx.doi.org/10.1016/j.jmbbm.2012.11.009. PMid:23262309.

3. Middleton, J. C., \& Tipton, A. J. (2000). Synthetic biodegradable polymers as orthopaedic devices. Biomaterials, 21(23), 2335 2346. http://dx.doi.org/10.1016/S0142-9612(00)00101-0. PMid:11055281.

4. Li, Z. H., Yu, A. X., Guo, X. P., Qi, B. W., Zhou, M., \& Wang, W. Y. (2013). Absorbable implants versus metal implants for the treatment of ankle fractures: A meta-analysis. Experimental and Therapeutic Medicine, 5(5), 1531-1537. http://dx.doi. org/10.3892/etm.2013.1017. PMid:23737914.

5. Hanawa, T. (2006). Recent development of new alloys for biomedical use. Materials Science Forum, 512, 243-248. http:// dx.doi.org/10.4028/www.scientific.net/MSF.512.243.

6. An, Y. H., Woolf, S. K., \& Friedman, R. J. (2000). Pre-clinical in vivo evaluation of orthopaedic bioabsorbable devices. Biomaterials, 21(24), 2635-2652. http://dx.doi.org/10.1016/ S0142-9612(00)00132-0. PMid:11071614.

7. Jarvela, S., Sihvonen, R., Sirkeoja, H., \& Jarvela, T. (2010). All- inside meniscal repair with bioabsorbable meniscal screws or with bioaborbable meniscus arrows A prospective, Randomized Clinical Study with 2-year results. The American
Journal of Sports Medicine, 38(11), 2211-2217. http://dx.doi. org/10.1177/0363546510374592. PMid:20716684.

8. Emond, C. E., Woelber, E. B., Kurd, S. K., Ciccotti, M. G., \& Cohen, S. B. (2011). A Comparison of the Results of Anterior Cruciate Ligament Reconstruction Using Bioabsorbable Versus Metal Interference Screws: A Meta-Analysis. The Journal of Bone \& Joint Surgery, 93(6), 572-580. http://dx.doi.org/10.2106/ JBJS.J.00269. PMid:21411708.

9. Hench, L. L., \& Ethridge, E. C. (1982). Biomaterials: an interfacial approach. New York: Academic Press.

10. Claes, L. E., Ignatius, A. A., Rehm, K. E., \& Scholz, C. (1996). New bioresorbable pin for the reduction of small bony fragments: design, mechanical properties and in vitro degradation. Biomaterials, 17(16), 1621-1626. http://dx.doi. org/10.1016/0142-9612(95)00327-4. PMid:8842367.

11. Törmälä, P., Vasenius, J., Vainionpaa, S., Laiho, J., Pohjonen, T., \& Rokkanen, P. (1991). Ultra-high-strength absorbable self-reinforced polyglycolide (SR-PGA) composite rods for internal fixation of bone fractures: in vitro and in vivo study. Journal of Biomedical Materials Research, 25(1), 1-22. http:// dx.doi.org/10.1002/jbm.820250102. PMid:1850429.

12. Kumar, A., \& Gupta, R. K. (1988). Fundamentals of polymers. New York: McGraw-Hill Companies, Inc.

13. Esposito, A. R., Moda, M., Cattani, S. M., Santana, G. M., Barbieri, J. A., Munhoz, M. M., Cardoso, T. P., Barbo, M. L., Russo, T., D’Amora, U., Gloria, A., Ambrosio, L., \& Duek, E. A. R. (2013). PLDLA/PCL-T Scaffold for meniscus tissue engineering. BioResearch Open Access, 2(2), 138-147. http:// dx.doi.org/10.1089/biores.2012.0293. PMid:23593566.

14. Motta, A. C., \& Duek, E. A. R. (2006). Síntese, caracterização e degradação "in vitro" do Poli(L-ácido láctico). Polímeros: Ciência e Tecnologia, 16(1), 26-32. http://dx.doi.org/10.1590/ S0104-14282006000100008.

15. Rezende, C. A., \& Duek, E. A. R. (2003). Poly (lactide-coglycolide) acid/poly (lactic acid) blends: in vitro degradation. Polímeros: Ciência e Tecnologia, 1(13), 36-44. http://dx.doi. org/10.1590/S0104-14282003000100009.

16. Luciano, R. M. (2001). Efeitos da concentração de plastificante em membranas de poli (Ácido Lático) para implantes (Tese de doutorado). Universidade Estadual de Campinas, Campinas.

17. Duarte, M. A., Duek, E. A. R., \& Motta, A. C. (2014). In Vitro Degradation of Poly (L-co-D,L lactic acid) Containing PCL-T. Polimeros: Ciência e Tecnologia, 1(24), 1-8. http://dx.doi. org/10.4322/polimeros.2014.056.

18. Saha, S. K., \& Tsuji, H. (2006). Effects of rapid crystallization on hydrolytic degradation and mechanical properties of poly(1-lactide-co- $\varepsilon$-caprolactone). Reactive \& Functional Polymers, 66(11), 1362-1372. http://dx.doi.org/10.1016/j. reactfunctpolym.2006.03.020.

19. Yuan, X., Mak, A. F. T., \& Yao, K. (2002). In vitro degradation of poly(L- lactic acid) fibers in phosphate buffered saline. Journal of Applied Polymer Science, 85(5), 936-943. http:// dx.doi.org/10.1002/app.10490.

20. Chapanian, R., Tse, M. Y., Pang, S. C., \& Amsden, B. (2010). Long term in vivo degradation and tissue response to photocross-linked elastomers prepared from star-shaped prepolymers of poly(epsilon-caprolactone-co-D,L-lactide). Journal of Biomedical Materials Research. Part A, 92A(3), 830-842. http://dx.doi.org/10.1002/jbm.a.32422. PMid:19280631. 